\title{
Mechanism of Effect of Thoracic Inferior Vena Cava Constriction on Renal Water Excretion
}

\author{
Robert J. Anderson, Pravit Cadnapaphornchat, Judith A. Harbottle, \\ Kerth M. McDonald, and Robert W. Schrier \\ From the Department of Medicine, University of Colorado Medical Center, \\ Denver, Colorado 80220
}

\begin{abstract}
A B S T R A C T Persistent secretion of vasopressin and/ or diminished distal fluid delivery have been proposed to explain the impaired water excretion associated with low-output cardiac failure. In the present investigation cardiac output (CO) was diminished in anesthetized dogs undergoing a water diuresis by constriction of the thoracic inferior vena cava (TIVC). In intact animals (group I) acute TIVC constriction decreased $\mathrm{CO}$ from 3.5 to 2.2 liters $/ \mathrm{min}(P<0.005)$ as urinary osmolality (Uosm) increased from 103 to 543 mosmols/ $\mathrm{kg}(P<0.001)$ and free water clearance $\left(\mathrm{C}_{\mathrm{H}_{2} \mathrm{O}}\right) \mathrm{de}-$ creased from 2.1 to $-0.6 \mathrm{ml} / \mathrm{min}(P<0.001)$. This antidiuretic effect was disassociated from changes in renal arterial and venous pressures, glomerular filtration rate, solute excretion, and renal innervation. To examine the role of vasopressin in this antidiuresis, studies.(group II) were performed in acutely hypophysectomized, steroid-replaced animals. In these animals TIVC constriction decreased $\mathrm{CO}$ to a similar degree from 3.4 to 2.1 liters $/ \mathrm{min}(P<0.001)$. However, the effects on $U_{\text {osm }}(87-104$ mosmols $/ \mathrm{kg})$ and $\mathrm{C}_{\mathrm{H}_{2} \mathrm{O}}$ (2.1$1.6 \mathrm{ml} / \mathrm{min}$ ) were significantly less than in intact dogs. In another group of hypophysectomized animals, (group III) renal arterial and venous pressures were not controlled, and the effect of TIVC constriction on $\mathrm{U}_{\mathrm{osm}}$ was not significant (65-79 mosmols $/ \mathrm{kg}$ ) although $\mathrm{C}_{\mathrm{H}_{2} \mathrm{O}}$ decreased from 3.3 to $1.9 \mathrm{ml} / \mathrm{min}(P<0.001)$. In both the group II and III studies, there were linear correlations between the changes in $\mathrm{C}_{\mathrm{H}_{2} \mathrm{O}}$ and the urine flow. Studies were also performed in baroreceptor-denervated animals with intact hypothalamo-neurohypophyseal tracts, and acute TIVC constriction altered neither Uosm nor $\mathrm{C}_{\mathrm{H}_{2} \mathrm{O}}$ when renal arterial pressure was controlled. These results therefore indicate that the effect of TIVC constriction on Uosm is primarily vasopressin
\end{abstract}

Dr. Anderson is a Teaching and Research Scholar of the American College of Physicians.

Received for publication 7 December 1973 and in revised form 15 August 1974. mediated while the effect on $\mathrm{C}_{\mathbf{E}_{2} \mathrm{O}}$ is mediated both by vasopressin release and diminished distal fluid delivery. A decrease in renal arterial pressure, or some consequence thereof, seems to be an important determinant of the latter effect.

\section{INTRODUCTION}

Acute constriction of the thoracic inferior vena cava (TIVC) ${ }^{1}$ has been used by numerous investigators $(1-4)$ as an experimental model of cardiac failure and is known to be associated with a diminished cardiac output ( $\mathrm{CO}$ ) (4) and increased tubular reabsorption of sodium (1-4). The effect of acute TIVC constriction on renal diluting capacity has not been previously investigated. An impairment in renal water excretion is, however, a frequent accompaniment of low-output cardiac failure (5). Although the mechanism of this defect in water excretion is unknown, it has been suggested to be mediated by either increased release of vasopressin (6) or diminished delivery of fluid to the distal diluting segment of the nephron (5). The present studies were therefore undertaken to examine the effect of acute TIVC constriction on renal water excretion and to define the mechanism of any such effect.

\section{METHODS}

40 experiments were performed on 24 mongrel dogs of either sex weighing $20-30 \mathrm{~kg}$. Food was withheld $18 \mathrm{~h}$ before the experiment and all animals had free access to water. The animals were anesthetized with i.v. pentobarbital $(30 \mathrm{mg} / \mathrm{kg})$ and intubated and ventilated with a Harvard respirator (Harvard Apparatus Co. Inc., Millis, Mass.). Nine animals underwent transbuccal hypophysectomy through the hard palate on the morning of the experiment (7). After induction of anesthesia, all animals received i.m. $5 \mathrm{mg}$ of deoxycorticosterone in oil. At this

${ }^{1}$ Abbreviations used in this paper: $\mathrm{C}_{\mathrm{H} 20}$, free water clearance; CO, cardiac output; GFR, glomerular filtration rate; $\mathrm{P}_{\text {osm }}$, plasma osmolality; RVR, renal vascular resistance; TIVC, thoracic inferior vena cava; $U_{o s m}$, urinary osmolality. 
time, each animal received an i.v. infusion of either $0.3 \%$ $\mathrm{NaCl}$ with $4 \mathrm{meq} / \mathrm{liter}$ of $\mathrm{KCl}$ or $2.5 \%$ dextrose at $10-15$ $\mathrm{ml} / \mathrm{min}$ which was continued throughout the experiment. TIVC constriction was produced either by placement of a snare around the TIVC or by inflation of a balloon placed in the TIVC via a femoral vein. TIVC constriction to a femoral venous pressure of $8-16 \mathrm{~mm} \mathrm{Hg}$ was performed because this degree of TIVC constriction has been previously demonstrated to result in a significant, reversible fall in CO (4). Renal venous pressure was controlled in 10 animals by a snare placed around the abdominal vena cava above the origins of both renal veins. In 17 animals, an adjustable Blalock clamp was placed around the aorta above both renal arteries to control renal arterial pressure. One kidney was denervated in 10 animals by stripping the nerves from the renal pedicle and applying 95\% alcohol. Carotid sinus denervation and bilateral cervical vagotomy were performed in four animals, as previously described (8). Polyethylene catheters were placed in both ureters and renal veins through bilateral flank incisions using a retroperitoneal approach. In all animals, catheters were placed in the aorta via the branchial artery for continuous measurement of arterial pressure and in the vena cava via the femoral vein to estimate renal venous pressure. In the animals with a Blalock clamp around the aorta, the arterial pressure was also measured in the aorta below the clamp via a catheter inserted in the femoral artery. All pressures were measured using Statham transducers (Statham Instruments, Inc., Oxnard, Calif.). Clearances of inulin and para-aminohippuric acid were measured by standard methods (9). The experiments were begun after a 1-2-h equilibration period when a hypotonic, stable urine flow had been obtained. During the experiments, urine was collected at 5-10-min intervals and arterial and renal vein samples were collected at the midpoint of alternate urine collections. $\mathrm{CO}$ was measured every other collection period by the dyedilution method (4). In some animals two consecutive experiments were performed. The experiments were carried out according to the following protocols :

Acute TIVC constriction in intact animals with constant renal arterial pressure-group I. In these experiments urine flow was allowed to stabilize, then three to five control periods were obtained after which acute TIVC constriction was performed. After a 30-40-min equilibration period, another three to five urine collection periods were obtained during continued TIVC constriction. Release of the TIVC constriction was followed by another 30-40-min equilibration period, then three to five post control urine collections were obtained. In all of these studies, renal arterial pressure was maintained constant by adjustment of the suprarenal aortic clamp, and in 8 of 13 experiments renal venous pressure was also maintained constant by adjustment of the abdominal vena cava snare. In three intact animals, dexamethasone ( $0.8 \mathrm{mg}$ i.m. and $0.8 \mathrm{mg}$ i.v.) was administered to insure that this glucocorticoid was not responsible for differences observed between intact and dexamethasone-treated (group II) animals.

Acute TIVC constriction in hypophysectomized animals with constant renal arterial pressure-group II. The studies in this group were performed in acutely hypophysectomized animals treated with dexamethasone $(0.8 \mathrm{mg}$ i.m. and $0.8 \mathrm{mg}$ i.v.) to eliminate any change in endogenous vasopressin during acute TIVC constriction. Otherwise, the protocol was the same as in the group I studies. Renal arterial and venous pressures were maintained constant in all experiments.
Acute TIVC constriction in hypophysectomized animals without constancy of renal venous and arterial pressuresgroup III. This study was performed to assess the extent to which failure to maintain constancy of renal arterial and venous pressure during acute TIVC constriction might enhance any vasopressin-independent effect.

The studies were, therefore, performed in steroid-replaced hypophysectomized animals. Otherwise, the protocol was the same as that used in the group I studies.

Acute TIVC constriction in animals with denervated baroreceptors-group IV. These studies were performed to examine whether intact parasympathetic pathways, namely glossopharyngeal and vagal pathways, are necessary for the antidiuretic effect of acute TIVC constriction. Renal arterial, but not renal venous, pressure was controlled in these studies. Other than using animals in which acute bilateral cervical vagotomy and carotid sinus denervation had been performed, the protocol was the same as in the group I studies. The analytical procedures used in these studies have been referred to elsewhere (9). Statistical analysis was performed by the analysis of variance for comparisons within groups and by the unpaired $t$ test for comparisons between groups. See footnote 1 for abbreviations used in this paper.

\section{RESULTS}

Effects of acute TIVC constriction in intact dogs with constant renal arterial pressure-group I (Table I a, Fig. 1). The results of these 14 studies in eight animals were no different whether renal venous pressure was controlled or not. Therefore, the results are presented together. An antidiuresis occurred in each experiment during acute TIVC constriction in these intact dogs undergoing a water diuresis. Acute TIVC constriction increased urinary osmolality (Uosm) from $103 \pm 7 \mathrm{SE}$ to $543 \pm 50$ mosmols $/ \mathrm{kg} \mathrm{H} \mathrm{H}_{2} \mathrm{O} \quad(P<0.001)$ and $\mathrm{U}_{\mathrm{osm}}$ fell to $177 \pm 29$ mosmols $/ \mathrm{kg} \mathrm{H}_{2} \mathrm{O}$ on release of the constriction (Fig. 1). This effect of TIVC constriction on $U_{\text {osm }}$ was accompanied by a decrease in free water clearance $\left(\mathrm{C}_{\mathrm{H}_{2} \mathrm{O}}\right)$ from $2.2 \pm 0.2$ to $-0.6 \pm 0.1$ $\mathrm{ml} / \mathrm{min}(P<0.001)$ which increased to $1.4 \pm 0.3 \mathrm{ml} /$ $\min (P<0.001)$ on release of the constriction. The mean $P_{o s m}$ did not increase significantly during acute TIVC constriction. The Posm's were $263 \pm 2,257 \pm 3$, and $252 \pm 3$ mosmols $/ \mathrm{kg}$ before, during, and after TIVC constriction, respectively.

This effect of acute TIVC constriction on renal water excretion was associated with a reversible decrease in $\mathrm{CO}(3.5 \pm 0.3$ to $2.2 \pm 0.3 \mathrm{liters} / \mathrm{min}, P<0.005$, to 3.7 $\pm 0.4 \pm 1$ liters $/ \mathrm{min}, P<0.005)$. Parallel changes in systemic arterial pressure occurred as renal arterial pressure was maintained constant. Glomerular filtration rate (GFR) was not altered by acute TIVC constriction, but renal vascular resistance (RVR) increased with constriction and returned to the control level on release of the constriction. The lowering of renal arterial pressure by aortic constriction before the control periods, may have increased tubular sodium reabsorption to a degree that antinatiuturesis during acute TIVC 
EFFECTS OF ACUTE THORACIC INFERIOR VENA CAVA CONSTRICTION

ON RENAL WATER EXCRETION IN DOGS UNDERGOING A WATER DIURESIS
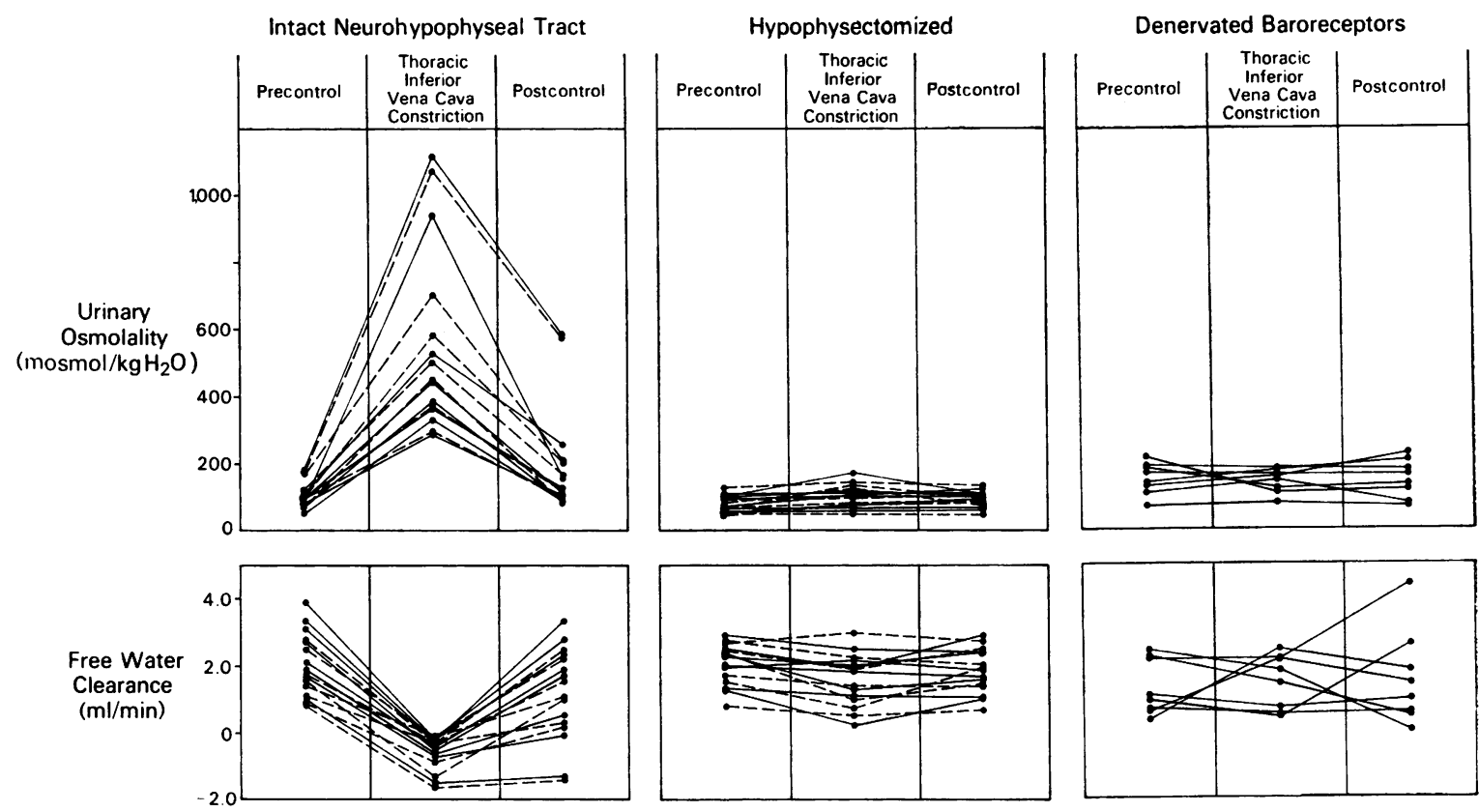

Figure 1 Effect of TIVC constriction on $U_{o s_{m}}$ (above) and $C_{H_{20}}$ (below) in intact (left), hypophysectomized (middle), and baroreceptor-denervated animals (right). The denervated and innervated kidneys are denoted by dashed and solid lines, respectively. Only the 8 experiments are plotted in the intact group in which renal venous and arterial pressures were maintained constant throughout the experiment.

constriction was not observed. There were no differences between the results in the innervated and denervated kidneys and, therefore, the data has been meaned in this and subsequent groups.

In six experiments performed in three dexamethasone-treated intact animals, TIVC constriction was associated with a significant, reversible fall in both $\mathrm{CO}$ (3.4 \pm 0.4 to $2.1 \pm 0.2$ liters $/ \mathrm{min}, P<0.005$, to $3.6 \pm 0.5$ liters $/ \mathrm{min}, P<0.025)$ and systemic arterial pressure $(140 \pm 7$ to $112 \pm 5 \mathrm{~mm} \mathrm{Hg}, P<0.005$, to $131 \pm 10 \mathrm{~mm}$ $\mathrm{Hg}, P<0.05)$. In these animals, renal venous and arterial pressures were kept constant at $12 \pm 0.2$ and $112 \pm 5 \mathrm{~mm} \mathrm{Hg}$, respectively. As in the intact animals, the nondexamethasone-treated animals' mean Uosm increased from $123 \pm 12$ to $701 \pm 104$ mosmols $/ \mathrm{kg} \mathrm{H} \mathrm{H}_{2} \mathrm{O}$ $(P<0.001)$ with TIVC constriction and fell to 186 \pm 26 mosmols $/ \mathrm{kg} \mathrm{H}_{2} \mathrm{O} \quad(P<0.001)$ on constriction release while $\mathrm{C}_{\mathrm{H}_{2} \mathrm{O}}$ decreased from $1.36 \pm 0.24$ to -0.659 $\pm 0.12 \mathrm{ml} / \mathrm{min}(P<0.001)$ with constriction and rose to $0.909 \pm 0.37 \mathrm{ml} / \mathrm{min}(P<0.001)$ on constriction release. GFR was not significantly altered by TIVC constriction ( $44 \pm 5$ to $46 \pm 6$ to $46 \pm 6)$. Neither RVR $(0.548 \pm 0.064$ to $0.679 \pm 0.090 \mathrm{~mm} \mathrm{Hg} / \mathrm{ml}$ per $\mathrm{min})$ nor the solute excretion rate $(285 \pm 28$ to $283 \pm 35$ mosmols/min) changed significantly with TIVC constric-

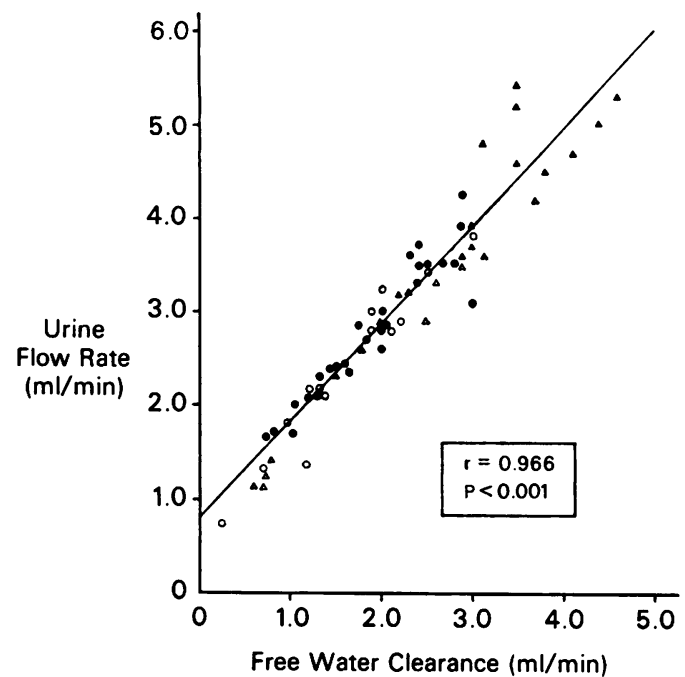

FIGURE 2 Linear correlation between urine flow rate and $\mathrm{C}_{\mathrm{H}_{2} \mathrm{O}}$ during control and TIVC constriction periods in hypophysectomized animals undergoing a water diuresis. The open and closed circles denote the results from the group II experiments while the open and closed triangles denote the results from group III experiments. In both groups, the open symbols represent the control collections while the closed symbols represent the experiment collections during TIVC constriction. 
TABLE I

Effect of Acute Constriction of the TIVC on Systemic and Renal Hemo-

\begin{tabular}{|c|c|c|c|c|c|c|c|c|c|}
\hline & \multicolumn{3}{|c|}{ Systemic arterial pressure } & \multicolumn{3}{|c|}{ Renal arterial pressure } & \multicolumn{3}{|c|}{ Renal venous pressure } \\
\hline & $\begin{array}{l}\text { Pre- } \\
\text { control }\end{array}$ & TIVC & $\begin{array}{l}\text { Post- } \\
\text { control }\end{array}$ & $\begin{array}{c}\text { Pre- } \\
\text { control }\end{array}$ & TIVC & $\begin{array}{l}\text { Post- } \\
\text { control }\end{array}$ & $\begin{array}{c}\text { Pre- } \\
\text { control }\end{array}$ & TIVC & $\begin{array}{l}\text { Post- } \\
\text { control }\end{array}$ \\
\hline (a) Group I $(n 14)^{*}$ & \multicolumn{3}{|c|}{$m m \mathrm{Hg}$} & \multicolumn{3}{|c|}{$m m \mathrm{Hg}$} & \multicolumn{3}{|c|}{$m m \mathrm{Hg}$} \\
\hline Mean & 154 & 125 & 150 & 124 & 123 & 123 & 10 & 12 & $10^{+}$ \\
\hline $\mathrm{SE}$ & \pm 5 & \pm 4 & \pm 4 & \pm 5 & \pm 5 & \pm 5 & \pm 1 & \pm 1 & \pm 1 \\
\hline$P$ value & \multicolumn{3}{|c|}{$<0.001 \quad<0.001$} & \multicolumn{2}{|c|}{ NS } & & \multicolumn{2}{|c|}{$<0.025$} & 25 \\
\hline \multicolumn{10}{|l|}{ (b) Group II ( $n 9)$} \\
\hline Mean & 140 & 117 & 134 & 115 & 117 & 116 & 12 & 13 & 13 \\
\hline $\mathrm{SE}$ & \pm 5 & \pm 4 & \pm 4 & \pm 4 & \pm 4 & \pm 4 & \pm 1 & \pm 1 & \pm 1 \\
\hline$P$ value & \multicolumn{3}{|c|}{$<0.001 \quad<0.001$} & \multicolumn{2}{|c|}{ NS } & & \multicolumn{2}{|c|}{$<0.025$} & \\
\hline \multicolumn{10}{|l|}{ (c) Group III ( $n 8$ ) } \\
\hline Mean & 139 & 111 & 138 & 139 & 111 & 138 & 6 & 12 & 6 \\
\hline $\mathrm{SE}$ & \pm 9 & \pm 8 & \pm 8 & \pm 9 & \pm 8 & \pm 8 & \pm 1 & \pm 1 & \pm 1 \\
\hline$P$ value & \multicolumn{3}{|c|}{$<0.001 \quad<0.001$} & \multicolumn{2}{|c|}{$<0.001$} & $<0.001$ & \multicolumn{3}{|c|}{$<0.001<0.001$} \\
\hline \multicolumn{10}{|l|}{ (d) Group IV ( $n$ 4) } \\
\hline Mean & 143 & 104 & 105 & 115 & 104 & 101 & 6 & 10 & 6 \\
\hline SE & \pm 12 & \pm 12 & \pm 11 & \pm 9 & \pm 12 & \pm 13 & \pm 2 & \pm 2 & \pm 2 \\
\hline$P$ value & \multicolumn{3}{|c|}{$<0.01$} & \multicolumn{2}{|c|}{ NS } & & \multicolumn{3}{|c|}{$<0.001<0.001$} \\
\hline
\end{tabular}

* Group I, intact dogs with renal arterial pressure controlled; group II, hypophysectomized dogs with renal arterial and renal venous pressure controlled; group III, hypophysectomized dogs without renal arterial and renal venous pressure controlled; group IV, baroreceptor denervated dogs with renal arterial pressure controlled; the values for renal hemodynamics and solute excretion are expressed for kidney.

$\ddagger 5$ of the 13 experiments were performed without renal venous pressure controlled.

tion. However, on constriction release, RVR significantly fell $(0.679 \pm 0.09$ to $0.515 \pm 0.069 \mathrm{~mm} \mathrm{Hg} / \mathrm{ml}$ per min, $P<0.025)$ and the solute excretion rate significantly rose $(283 \pm 35$ to $418 \pm 31$ mosmols $/ \mathrm{min}, P<$ 0.005 ). Hence, a similar antidiuresis with TIVC constriction was observed in both intact animals with and without exogenous dexamethasone.

Effects of acute TIVC constriction in hypophysectomized dogs with constant renal arterial pressuregroup II (Table I b, Figs. 1 and 2). In this group nine studies were performed on five dogs to examine whether any of the antidiuresis observed during acute TIVC constriction is due to increased release of endogenous vasopressin. In contrast to intact animals, acute constriction of the TIVC in acutely hypophysectomized, steroid-replaced animals was not associated with a profound antidiuresis. The mean $U_{o s m}$ increased slightly from $87 \pm 5$ to $104 \pm 9$ mosmols $/ \mathrm{kg} \mathrm{H}_{2} \mathrm{O} \quad(P<$ 0.01 ) during TIVC constriction and decreased to 94 \pm 6 mosmols $/ \mathrm{kg} \mathrm{H}_{2} \mathrm{O}(P<0.1)$ on release of the constriction. During the same periods $\mathrm{C}_{\mathrm{H}_{2} \mathrm{O}}$ decreased from $2.1 \pm 0.2$ to $1.6 \pm 0.2 \mathrm{ml} / \mathrm{min}(P<0.005)$ and increased to $1.8 \pm 0.2 \mathrm{ml} / \mathrm{min}(P<0.1)$. The plasma osmolality ( $P_{\text {osm }}$ ) before, during, and after TIVC constriction were $274 \pm 4,270 \pm 4$, and $269 \pm 4$ mosmols/kg, respectively, and were not significantly different. The marked differences in the effect of acute TIVC on $U_{o s m}$ and $\mathrm{C}_{\mathrm{H}_{2} \mathrm{O}}$ in the intact (group I) and hypophysectomized (group II) dogs are shown in Fig. 1. The modest antidiuretic effect of TIVC constriction in hypophysectomized dogs was examined in relationship to distal delivery of fluid to the diluting segment (Fig. 2). In the absence of vasopressin, the linear correlation between urine flow and $\mathrm{C}_{\mathrm{H}_{2} \mathrm{O}}$ during both control and TIVC constriction periods suggests that any variation in these parameters was due to changes in distal fluid delivery out of the proximal tubule. Values from the group III experiments in which renal venous and arterial pressure were not controlled are also plotted in Fig. 2 and demonstrated the same phenomenon.

The effects on systemic and renal hemodynamics in the intact and hypophysectomized animals were quite similar (Table I $a$ and $b$ ), although the effects on $U_{\text {osm }}$ and $\mathrm{C}_{\mathrm{H}_{2} \mathrm{O}}$ were substantially different (Fig. 1). Acute TIVC constriction in the hypophysectomized dogs was associated with a decrease in CO from $3.4 \pm 0.3$ to $2.1 \pm 0.2$ liters $/ \mathrm{min} \quad(P<0.001)$ which returned to $3.0 \pm 0.2$ liters $/ \mathrm{min}(P<0,005)$. Parallel changes in arterial 
dynamics and Solute Excretion in Dogs undergoing a Water Diuresis

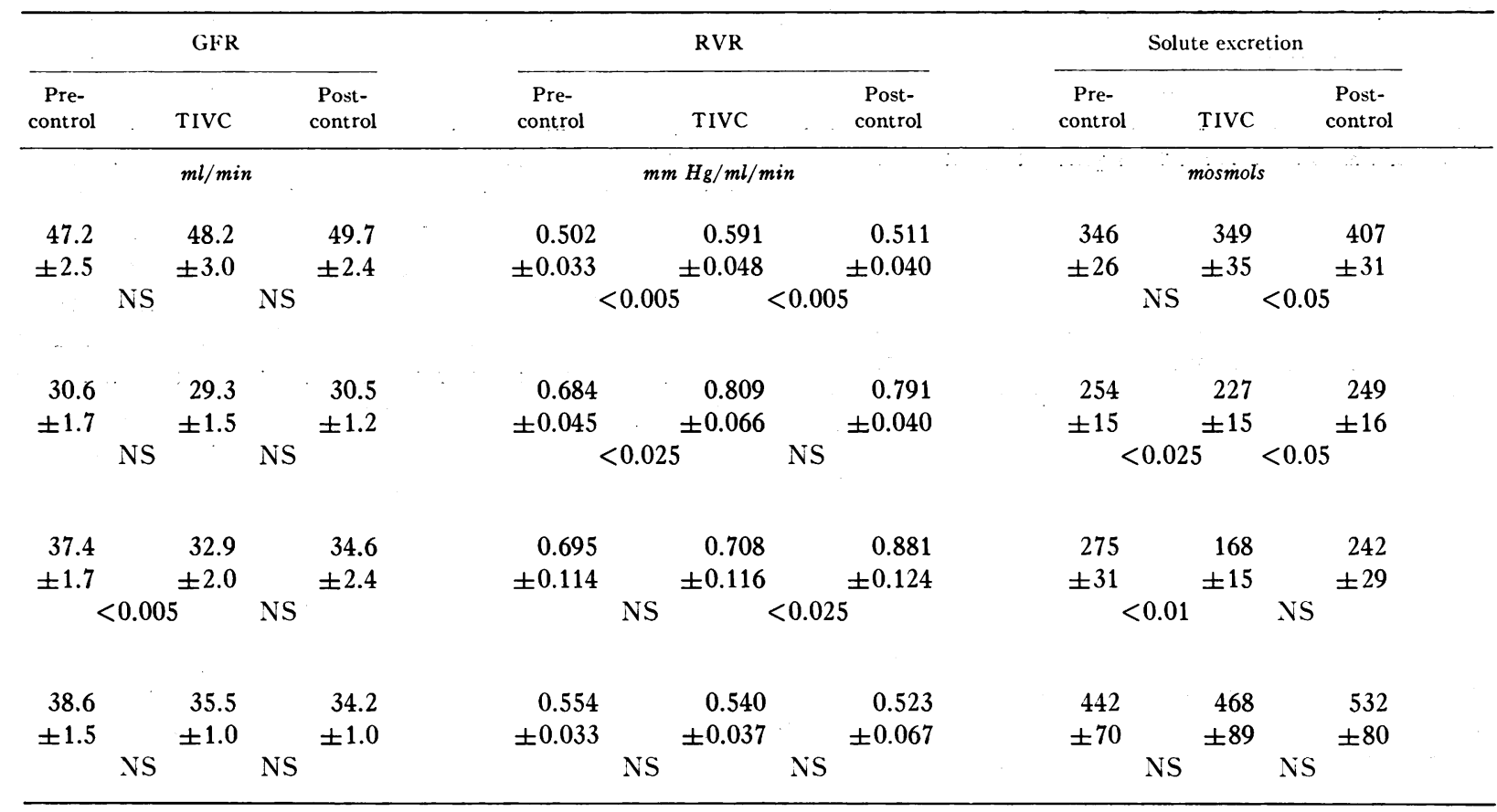

pressure also occurred. GFR remained constant but RVR increased during TIVC constriction but did not decrease significantly on release of the constriction. In contrast to the intact dogs, the hypophysectomized animals demonstrated a significant decrease in solute excretion, which increased insignificantly on release of the constriction.

Effect of acute TIVC constriction in hypophysectomized animals without constancy of renal venous or arterial pressure-group III (Table I c, Fig. 2). In this group eight experiments were performed on four animals to delineate the degree of effect of acute TIVC constriction on water excretion in hypophysectomized animals when renal arterial and venous pressure are not controlled. In these animals, TIVC constriction was associated with an insignificant increase in $\mathrm{U}_{\mathrm{s} m \mathrm{~m}}$ from $65 \pm 7$ to $79 \pm 11$ mosmols $/ \mathrm{kg} \mathrm{H} \mathrm{H}_{2} \mathrm{O}$ which fell to $67 \pm 6$ mosmols $/ \mathrm{kg} \mathrm{H} \mathrm{H}_{2} \mathrm{O}$ on release of the constriction. With TIVC constriction, $\mathrm{C}_{\mathbf{B}_{2} \mathrm{O}}$ fell significantly from $3.3 \pm 0.2$ to $1.9 \pm 0.3 \mathrm{ml} / \mathrm{min}(P<0.001)$ and increased to 2.8 $\pm 0.3 \mathrm{ml} / \mathrm{min}(P<0.005)$ on release of the constriction. The decrease in $\mathrm{C}_{\mathrm{H}_{2} \mathrm{O}}$ during TIVC constriction in the group III studies was significantly greater than those changes observed with TIVC constriction in hy- pophysectomized animals in which renal venous and renal arterial pressure were controlled (group II). There were no significant differences in $\mathrm{P}_{\text {osm }}$ before during, or after $(279 \pm 3,278 \pm 4$, or $277 \pm 5$ mosmols/ $\mathrm{kg} \mathrm{H}_{2} \mathrm{O}$ ) TIVC constriction in these group III studies. As in all other studies, TIVC constriction was associated with a significant fall in mean systemic arterial pressure as parallel changes in renal venous and arterial pressure were allowed to occur. There was also a significant fall in GFR during the TIVC constriction. Effects of acute TIVC constriction in baroreceptor denervated animals-group IV (Table Id, Fig. 1). In this group four studies were performed on four animals to examine whether changes in autonomic neural tone in parasympathetic pathways are responsible for vasopressin release with TIVC constriction. In these animals acute TIVC constriction decreased cardiac output from $4.0 \pm 1.4$ to $2.9 \pm 0.9$ liters $/ \mathrm{min} \quad(P<0.001)$ which increased to $4.0 \pm 0.9$ liters $/ \mathrm{min}(P<0.001)$ on release of the TIVC constriction. No antidiuresis was observed in these studies as the Uosm's were $151 \pm 17$, $145 \pm 12$, and $150 \pm 21$ mosmols $/ \mathrm{kg} \mathrm{H}_{2} \mathrm{O}$ before, during, and after TIVC constriction, respectively. During the same periods $\mathrm{C}_{\mathrm{H}_{2} \mathrm{O}}$ was $1.26 \pm 0.3,1.42 \pm 0.3$, and 1.55 
$\pm 0.5 \mathrm{ml} / \mathrm{min}$ before, during, and after TIVC constriction, respectively. The mean Posm's before, during, and after (252 \pm 5 to $250 \pm 4$ to $248 \pm 5$ mosmols $/ \mathrm{kg} \mathrm{H}_{2} \mathrm{O}$ ) TIVC constriction were not significantly different. In these studies, TIVC constriction was associated with a decrease in $\mathrm{CO}$ and systemic arterial pressure while GFR and RVR were not significantly altered.

\section{DISCUSSION}

Low-output cardiac failure is known to be associated with an impaired capacity to excrete solute-free water $(5,6)$. The mechanism involved in this disorder of water metabolism has not been defined. However, an effect mediated by persistent secretion of vasopressin (6) or an intrarenal effect mediated by diminished delivery of tubular fluid to the distal diluting segment of the nephron (5) have been proposed. In the present study, acute constriction of the inferior vena cava was used as a model of cardiac failure to further examine the effect of diminished cardiac output on renal water excretion. Several groups of experiments were performed to delineate the factors which might alter renal water excretion as $\mathrm{CO}$ is diminished.

In 8 of the 12 initial studies (group I), the effect of acute TIVC constriction on water excretion was examined in animals in which renal arterial and venous pressure were not allowed to change. Since changes in both renal arterial (10) and venous pressure (11) are known to affect tubular fluid reabsorption, it seemed important to examine whether acute TIVC constriction affects water excretion independent of these intrarenal hemodynamic alterations. In each Group I experiment performed in dogs undergoing a water diuresis, acute TIVC constriction diminished $\mathrm{CO}$ and caused an antidiuresis. This antidiuresis occurred in the absence of significant changes in glomerular filtration and solute excretion, two known determinants of renal water excretion $(12,13)$. RVR, however, did increase during acute TIVC constriction in these intact animals, thus intrarenal hemodynamic factors could not be excluded as contributing to the antidiuretic response (14). Even so, the profound increase in Uosm (103 \pm 7 to $543 \pm 50 \mathrm{mosmols} / \mathrm{kg}$ ) which occurred in association with this modest increase in renal vascular resistance, suggested that increased release of vasopressin could be a primary mediator of the antidiuretic response.

Studies therefore were performed in acutely hypophysectomized, steroid-replaced dogs undergoing a water diuresis. Such hypophysectomized animals may at times have lower mean arterial blood pressures and filtration rates than intact animals, but these animals nevertheless have been adequately shown to respond to exogenous vasopressin (15). This model has therefore proved very useful in delineating the role of en- dogenous vasopressin in various circumstances in which renal water excretion may be altered, such as during alpha (15) and beta (17) adrenergic stimulation, bilateral cervical vagotomy (16), angiotensin (17), nicotine administration (18), or atrial tachycardia (19), In the present study, acute TIVC constriction was found to have only a slight effect on Uosm (87 55 to $104 \pm 9$ mosmols $/ \mathrm{kg}$ ) in animals in which the source of vasopressin had been removed. Even this modest effect was not significantly reversible. Similarly, $\mathrm{C}_{\mathrm{H}_{2} \mathrm{O}}$ decreased only modestly during acute TIVC constriction in these hypophysectomized animals (group II) as compared to the intact animals (group I). These results therefore lend support to the conclusion that increased release of vasopressin is a primary factor in the effect of acute TIVC constriction on renal water excretion. The possibility seemed likely, however, that the vasopressinindependent effect of acute TIVC constriction on renal water excretion might be more pronounced in the absence of control of renal arterial and venous pressure. This possibility was tested in the group III experiments in hypophysectomized animals in which renal arterial pressure fell and renal venous pressure rose during acute TIVC constriction. The resultant decrement in $\mathrm{C}_{\mathrm{H}_{2} \mathrm{O}}$ was indeed most pronounced in this group of experiments. The effect of acute TIVC constriction to increase profoundly $\mathrm{U}_{\text {osm }}$ in intact animals, however, was not duplicated in this group of hypophysectomized, steroid-replaced animals.

Taken together, therefore, the present results indicate that both intrarenal factors and vasopressin release are involved in the effect of acute TIVC constriction to impair the renal capacity to excrete solute-free water. The vasopressin-dependent component of this antidiuretic effect appears to be primarily responsible for the osmotic water movement in the distal nephron and thus affects both $U_{0 s m}$ and $\mathrm{C}_{\mathrm{H}_{2} \mathrm{O}}$. Analysis of the vasopressin-independent component of the effect of acute TIVC on water excretion (groups II and III) demonstrated a linear correlation between changes in urine flow and $\mathrm{C}_{\mathrm{H}_{2} \mathrm{O}}$ (Fig. 2). These results therefore indicate that the alterations in intrarenal factors during acute TIVC constriction diminish renal water excretion by altering the rate of tubular fluid delivery to the distal nephron. This diminished distal fluid delivery, however, was not of a sufficient degree to profoundly influence $U_{\text {osm }}$.

Further studies (group IV) were also undertaken in the present investigation to examine the pathway whereby acute TIVC constriction stimulates vasopressin release. The results of recent studies from our laboratory $(8,16,20)$ prompted us to examine whether intact parasympathetic pathways are necessary to demonstrate the effect of acute TIVC on renal water ex- 
cretion. Renal arterial, but not venous, pressure was kept constant in these experiments. In these group IV experiments, acute TIVC constriction was found not to significantly alter $U_{\text {osm }}$ or free water excretion. The modest rise in renal venous pressure in these experiments during acute TIVC constriction, thus does not seem to alter renal water excretion. More importantly, the results also suggest that acute TIVC constriction stimulates vasopressin release by altering parasympathetic neural tone. In this regard, this effect of acute TIVC constriction to increase vasopressin release is similar to the effect of beta adrenergic stimulation (17). A similar alteration in renal water excretion can also be found to occur as arterial pressure at the level of the carotid sinus is diminished by bilateral carotid constriction to a similar degree as observed during acute TIVC constriction and beta adrenergic stimulation (19). Changes in systemic arterial pressure thus appear to affect renal water excretion by two independent pathways. On the one hand, an alteration in pressure at the level of the arterial baroreceptors affects parasympathetic afferent tone which in turn modulates vasopressin release. At the same time, the concomitant change in renal arterial pressure affects renal water excretion primarily by altering the rate of distal fluid delivery to the diluting segment of the nephron. Both of these mechanisms are involved in the effect of acute TIVC constriction, and possibly low-output cardiac failure, on renal water excretion.

It should be noted that acute TIVC constriction as a model of low-output cardiac failure, may not be completely analogous to low-output failure occurring in man with left ventricular disease in which the accompanying rise in left atrial pressure may activate left atrial receptors and antagonize baroreceptor stimulated antidiuretic hormone secretion (20). However, in the hyponatremic patient with low-output cardiac failure, it is likely that the antidiuretic hormone-inhibiting effect of left atrial distension is overidden by baroreceptor-mediated antidiuretic hormone release.

\section{ACKNOWLEDGMENTS}

We wish to express our gratitude to Ms. Diana LaRocco for excellent secretarial assistance and $\mathrm{Mr}$. Lowell Gilbert and Miss Abby McCool for excellent technical assistance.

These studies were supported by grants HL 15467-01 and HL 15629-01 from the National Institutes of Health, a grant from the Hoechst Pharmaceutical company, and a grant from the American College of Physicians.

\section{REFERENCES}

1. Cirksena, W. J., J. H. Dirks, and R. W. Berliner. 1966. Effect of thoracic cava obstruction on response of proximal tubule sodium reabsorption to saline infusion. J. Clin. Invest. 45: 179-186.

2. Earley, L. E., J. A. Martino, and R. M. Friedler. 1966. Factors affecting sodium reabsorption by the proximal tubule as determined during blockade of distal sodium reabsorption. J. Clin. Invest. 45: 1668-1684.

3. Levinsky, N. G., and R. C. Lalone. 1965. Sodium excretion during acute saline loading in dogs with vena cava constriction. J. Clin. Invest. 44: 565-573.

4. Schrier, R. W., and M. H. Humphreys. 1971. Factors involved in the antinatriuretic effects of acute constriction of the thoracic and abdominal inferior vena cava. Circ. Res. 29 : 479-489.

5. Bell, N. H., H. P. Schedl, and F. C. Bartter. 1964. An explanation for abnormal water retention and hypoosmolality in congestive heart failure. Am. J. Med. 36: 351-360.

6. Leaf, A., and A. R. Mamby. 1952. An antidiuretic mechanism not regulated by extracellular fluid tonicity. J. Clin. Invest. $31: 60-71$.

7. Schrier, R. W., R. Lieberman, and R. C. Ufferman. 1962. Mechanism of antidiuretic effect of beta adrenergic stimulation. J. Clin. Invest. 51: 97-111.

8. Berl, T., P Cadnapaphornchai, J. A. Harbottle, and R. W. Schrier. 1974. Mechanism of suppression of vasopressin during alpha-adrenergic stimulation with norepinephrine. J. Clin. Invest. 53: 219-227.

9. Schrier, R. W., and L. E. Earley. 1970. Effects of hematocrit on renal hemodynamics and sodium excretion in hydropenic and volume-expanded dogs. J. Clin. Invest. 49: 1656-1667.

10. Thurau, K., and P. Deetjen. 1962. Die Diurese bie arteriellen Drucksteigerungen: Bedeutung der Hamodynamik des Nierenmarkes fur die Harnkonzentrierung. Pfluegers Archiv. Gesamte Physiol. Menschen Tiere. 274: $567-580$.

11. Wathen, R. L., and E. E. Selkurt. 1969. Intrarenal regulatory factors of salt excretion during renal venous pressure elevation. Am. J. Physiol. 216: 1517-1524.

12. Levinsky, N. G., D. D. Davidson, and R. W. Berliner. 1959. Effects of reduced glomerular filtration on urine concentration in the presence of antidiuretic hormone. J. Clin. Invest. 38 : 730-740.

13. Wesson, L. G., Jr., and W. P. Anslow, Jr. 1948. Excretion of sodium and water during osmotic diuresis in the dog. Am. J. Physiol. 153: 465-474.

14. Martino, J. A., and L. E. Earley. 1967. Demonstration of a role of physical factors as determinants of the natriuretic response to volume expansion. J. Clin. Invest. 46: 1963-1978.

15. Schrier, R. W., and T. Berl. 1973. Mechanism of effect of alpha adrenergic stimulation with norepinephrine on renal water excretion. J. Clin. Invest. 52: 502-509.

16. Schrier, R. W., and T. Berl. 1972. Mechanism of the antidiuretic effect associated with interruption of parasympathetic pathways. J. Clin. Invest. 51: 2613-2620.

17. Cadnapaphornchai, P., J. Boykin, J. A. Harbottle, K M. McDonald, and R. W. Schrier. Effect of angiotensin II on renal water excretion. Am. J. Physiol. In press.

18. Cadnapaphornchai, P., J. L. Boykin, T. Berl, K. M McDonald, and R. W. Schrier. Mechanism of effect of nicotine on renal water excretion. Am. J. Physiol. In press.

19. Berl, T., P. Cadnapaphornchai, J. A. Harbottle, and R. W. Schrier. 1974. Mechanism of stimulation of vasopressin release during beta-adrenergic stimulation with isoproterenol. J. Clin. Invest. 53 : 857-867.

20. Boykin, J., P. Cadnapaphornchai, T. Burke, K. M. McDonald, and R. W. Schrier. 1974. Mechanism of the diuretic effect of atrial tachycardia. Clin. Res. 22: 265 a. (Abstr.) 